\title{
MORNING CHRONOTYPE IS A PROTECTIVE FACTOR \\ AGAINST CHEMOTHERAPY-INDUCED HOT FLASHES IN PREMENOPAUSAL WOMEN WITH BREAST CANCER
}

Kyung-Lak Son ${ }^{1}$, Dooyoung Jung2, Kwang-Min Lee ${ }^{3}$, Heesung Hwang ${ }^{1}$, JooYoung Lee ${ }^{4}$ Tae-Yong Kim ${ }^{5}$ Seock-Ah Im $^{5}$, Kyung-Hun Lee ${ }^{5}$, Bong-Jin Hahm ${ }^{1,3}$

Seoul National University Hospital, Neuropsychiatry, Seoul, Republic of Korea.

Ulsan National Institute of Science and Technology, Human Factors Engineering, Ulsan, Republic of Korea. ${ }^{3}$ Seoul National University College of Medicine, Psychiatry and Behavioral Sciences, seoul, Republic of Korea. 'Armed Forces Medical Command, Health Management, Seongnam, Republic of Korea.

SSeoul National University Hospital, Internal Medicine, Seoul, Republic of Korea.

\section{OBJECTIVE}

- Adjuvant chemotherapy in patients with breast cancer often causes hot flashes, impairing quality of life. However, the psychiatric factors associated with the development of chemotherapy-induced hot flashes (CIHFs) remain undetermined.

- The purpose of this study was to investigate whether chronotype was associated with the incidence of CIHFs.

\section{METHODS}

- A total of 119 premenopausal women with early-stage breast cancer awaiting adjuvant chemotherapy after surgery without hot-flashes were included.

- The presence of CIHF was defined as having moderate-to-high level of hot flashes symptom by the hot flashes subscale in the Menopause Rating Scale, at 6 months after the completion of chemotherapy.

- Chronotype (morning/intermediate/evening) was assessed with the Composite Scale of Morningness before adjuvant chemotherapy.

- To examine the association between chronotype and CIHF, we built logistic regression models, adjusting for age, body mass index, sleep quality, and radiation therapy.

\section{RESULTS}

\begin{tabular}{|c|c|c|c|}
\hline Characteristics & $\begin{array}{c}\text { Normal }(n=59) \\
\text { Mean } \pm \text { SD or } N(\%)\end{array}$ & $\begin{array}{l}\text { Hot-flashes }(n=60) \\
\text { Mean } \pm \text { SD or N }(\%)\end{array}$ & $p$ value \\
\hline Age & $43.1 \pm 5.7$ & $43.1 \pm 6.1$ & 0.964 \\
\hline Age $\geq 50$ years & $9(15.3 \%)$ & $13(21.7 \%)$ & 0.506 \\
\hline $\mathrm{BMl}, \mathrm{kg} / \mathrm{m} 2$ & $22.4 \pm 2.7$ & $22.5 \pm 2.9$ & 0.866 \\
\hline $\mathrm{BMl} \geq 25 \mathrm{~kg} / \mathrm{m} 2$ & $17(30.9 \%)$ & $24(40.7 \%)$ & 0.373 \\
\hline Married & $48(81.4 \%)$ & $46(76.7 \%)$ & 0.687 \\
\hline College graduates or higher & 34 (57.6\%) & $36(60.0 \%)$ & 0.939 \\
\hline Employment & & & 0.713 \\
\hline Homemaker & $30(50.8 \%)$ & $27(45.8 \%)$ & \\
\hline Work place & $29(49.2 \%)$ & $32(54.2 \%)$ & \\
\hline One alcoholic drinks per week & $10(16.9 \%)$ & $14(23.3 \%)$ & 0.523 \\
\hline History of smoking & $6(10.2 \%)$ & $4(6.7 \%)$ & 0.720 \\
\hline Disease stage & & & 0.269 \\
\hline 1 & $18(30.5 \%)$ & $21(35.0 \%)$ & \\
\hline ॥ & $33(55.9 \%)$ & $36(60.0 \%)$ & \\
\hline III & $8(13.6 \%)$ & $3(5.0 \%)$ & \\
\hline Operation & & & 0.150 \\
\hline $\mathrm{BCS}$ & $36(61.0 \%)$ & $45(75.0 \%)$ & \\
\hline $\mathrm{TM}$ & $23(39.0 \%)$ & $15(25.0 \%)$ & \\
\hline Radiation therapy & $41(69.5 \%)$ & $51(85.0 \%)$ & 0.072 \\
\hline Chemotherapy & & & 0.935 \\
\hline AC.D & 27 (45.8\%) & $26(43.3 \%)$ & \\
\hline FAC & $32(54.2 \%)$ & $34(56.7 \%)$ & \\
\hline Hormone therapy & 45 (76.3\%) & 45 (75.0\%) & 1.000 \\
\hline Chronotype & & & 0.067 \\
\hline Intermediate & $11(18.6 \%)$ & $16(26.7 \%)$ & \\
\hline Morning & $26(44.1 \%)$ & $33(55.0 \%)$ & \\
\hline Evening & $22(37.3 \%)$ & $11(18.3 \%)$ & \\
\hline PSQI & $5.0 \pm 2.9$ & $6.6 \pm 3.8$ & 0.014 \\
\hline Good sleep & $51(89.5 \%)$ & $44(75.9 \%)$ & 0.093 \\
\hline Poor sleep & $6(10.5 \%)$ & $14(24.1 \%)$ & \\
\hline HADS-D & $5.8 \pm 3.8$ & $6.8 \pm 3.5$ & 0.134 \\
\hline Not depressed & 39 (66.1\%) & 39 (65.0\%) & 1.000 \\
\hline Depressed & $20(33.9 \%)$ & $21(35.0 \%)$ & \\
\hline HADS-A & $5.6 \pm 3.3$ & $6.5 \pm 3.8$ & 0.159 \\
\hline Not anxious & $42(71.2 \%)$ & $42(70.0 \%)$ & 1.000 \\
\hline Anxious & $17(28.8 \%)$ & $18(30.0 \%)$ & \\
\hline
\end{tabular}

Abbreviations: AC-D, adriamycin/cyclophosphamide and docetaxel; BCS, breast-conserving surgery; BMI, body mass index; FAC, fluorouracil/adriamycin/cyclophosphamide; HADS, Hospital Anxiety and Depression Scale (HADS-D and HADS-A: subscales for depression and anxiety, respectively); PSQI, Pittsburgh Sleep Quality Index; TM, total mastectomy

\begin{tabular}{|c|c|c|}
\hline \multirow{2}{*}{ Factors } & \multicolumn{2}{|c|}{ Univariate analyses } \\
\hline & OR $(95 \% \mathrm{Cl})$ & $p$ value \\
\hline \multicolumn{3}{|l|}{ Age at baseline } \\
\hline$<50$ years & 1.00 (reference) & \\
\hline$\geq 50$ years & $1.54(0.61-4.04)$ & 0.370 \\
\hline \multicolumn{3}{|l|}{ BMl, kg/m2 } \\
\hline$<23$ kg/m2 & 1.00 (reference) & \\
\hline$\geq 23$ kg/m2 & $1.53(0.71-3.36)$ & 0.279 \\
\hline \multicolumn{3}{|l|}{ Stage of disease } \\
\hline I & 1.00 (reference) & \\
\hline II & $0.94(0.42-2.06)$ & 0.867 \\
\hline III & $0.32(0.06-1.30)$ & 0.130 \\
\hline \multicolumn{3}{|l|}{ Operation } \\
\hline BCS & 1.00 (reference) & \\
\hline TM & $0.52(0.23-1.13)$ & 0.104 \\
\hline \multicolumn{3}{|l|}{ Radiation therapy } \\
\hline No & 1.00 (reference) & \\
\hline Yes & $2.49(1.03-6.35)$ & 0.047 \\
\hline \multicolumn{3}{|l|}{ Chemotherapy } \\
\hline AC.D & 1.00 (reference) & \\
\hline FAC & $1.10(0.53-2.28)$ & 0.790 \\
\hline \multicolumn{3}{|l|}{ Hormone Therapy } \\
\hline No & 1.00 (reference) & \\
\hline Yes & $0.93(0.40-2.16)$ & 0.872 \\
\hline \multicolumn{3}{|l|}{ Chronotype } \\
\hline Intermediate & 1.00 (reference) & \\
\hline Morning & $0.37(0.16-0.94)$ & 0.040 \\
\hline Evening & $1.15(0.46-2.94)$ & 0.773 \\
\hline \multicolumn{3}{|l|}{ Sleep quality } \\
\hline Good & 1.00 (reference) & \\
\hline Poor & $2.70(0.99-8.19)$ & 0.060 \\
\hline \multicolumn{3}{|l|}{ Depression } \\
\hline Not depressed & 1.00 (reference) & \\
\hline Depressed & $1.05(0.49-2.25)$ & 0.899 \\
\hline \multicolumn{3}{|l|}{ Anxiety } \\
\hline Not anxious & 1.00 (reference) & \\
\hline Anxious & $1.06(0.48-2.34)$ & 0.887 \\
\hline
\end{tabular}

Table 3. Multivariate analyses of predictors for chemotherapy induced hot-flashes

\begin{tabular}{|c|c|c|}
\hline \multirow{2}{*}{ Factors } & \multicolumn{2}{|c|}{ Multivariate analyses } \\
\hline & OR $(95 \% \mathrm{Cl})$ & $p$ value \\
\hline \multicolumn{3}{|l|}{ Age at baseline } \\
\hline$<50$ years & 1.00 (reference) & \\
\hline$\geq 50$ years & $2.58(0.82-9.07)$ & 0.117 \\
\hline \multicolumn{3}{|l|}{ BMl, kg/m2 } \\
\hline$<23 \mathrm{~kg} / \mathrm{m} 2$ & 1.00 (reference) & \\
\hline$\geq 23 \mathrm{~kg} / \mathrm{m} 2$ & $1.45(0.60-3.55)$ & 0.406 \\
\hline \multicolumn{3}{|c|}{ Radiation therapy } \\
\hline No & 1.00 (reference) & \\
\hline Yes & $2.69(0.99-7.93)$ & 0.060 \\
\hline \multicolumn{3}{|l|}{ Chronotype } \\
\hline Intermediate & 1.00 (reference) & \\
\hline Morning & $0.37(0.13-0.96)$ & 0.045 \\
\hline Evening & $1.23(0.44-3.54)$ & 0.692 \\
\hline \multicolumn{3}{|l|}{ Sleep quality } \\
\hline Good & 1.00 (reference) & \\
\hline Poor & $1.95(0.62-6.87)$ & 0.060 \\
\hline
\end{tabular}

- CIHF occurred in $\mathbf{5 0 . 4 \%}$ of participants.

- The morning type was inversely associated with CIHF (reference: intermediate type, odds ratio [OR], $0.37 ; 95 \%$ confidence interval $[\mathrm{Cl}], 0.16-0.94 ; \mathrm{p}=0.040$ ) in the univariate model.

- The association remained significant $(\mathrm{OR}, 0.37 ; \mathrm{Cl}, 0.13-0.96 ; \mathrm{p}=$ 0.045 ) after adjusting for age, body mass index, sleep quality, and radiation therapy.

\section{Discussion}

- The morning chronotype is a protective factor against the development of CIHF in patient with breast cancer.

- Chronotypes should be assessed and considered in the prediction and management of CIHF.

\section{CONTACT INFO}

Kyung-Lak Son, M.D.

Research Fellow, Department of Neuropsychiatry,

Seoul National University Hospital, Seoul, Korea

E-mail: klson01@gmail.com 